\title{
Circulating secretory component in breast neoplasms
}

\author{
D KVALE, T O ROGNUM, * E THORUD, $†$ S D FOSSÅ, $†$ J S R $\varnothing, \ddagger$ P BRANDTZAEG \\ From the Laboratory for Immunohistochemistry and Immunopathology, Institute of Pathology, and *Institute of \\ Forensic Medicine, The National Hospital, Rikshospitalet, $\dagger$ The Norwegian Radium Hospital, and $\ddagger$ The \\ Norwegian Lutheran Hospital, University of Oslo, Oslo, Norway
}

SUMMARY The serum concentrations of IgAp and IgMr associated secretory component ( $\operatorname{IgA}$ and SIgM) of 98 patients with neoplasms of the breast were measured. Of the 56 patients with carcinomas, 11 had increased concentrations of circulating SIgM, which was almost twice as sensitive as SIgA as a marker for carcinoma. Concentrations of circulating SIgA and SIgM were independent of expression of secretory component, IgA, and carcinoembryonic antigen (CEA); histological tumour grade; and tumour cell DNA ploidy, whereas a weak correlation between SIgA and SIgM and circulating CEA was seen. The three patients who had liver metastases indicated had particularly high concentrations of circulating SIgA and SIgM, whereas no difference was generally seen between patients with malignancy and those with benign tumours.

Secretory component is derived from a transmembrane glycoprotein, ${ }^{1}$ expressed by secretory epithelial cells. Secretory component functions as a receptor facilitating the transport of polymeric immunoglobulins (pIgA and pIgM) containing $J$ chain into exocrine fluids ${ }^{2}$, where it partly remains bound to the secretory immunoglobulins (SIgA and SIgM) and partly appears as excess free secretory component. ${ }^{3}$ Human lactating mammary glands contain an active secretory immune system and considerable amounts of secretory component and $\operatorname{SIgA}$ are stored in the duct system. ${ }^{4}$

Neoplastic secretory epithelium may synthesise secretory component, both in vitro ${ }^{3}$ and in vivo, ${ }^{5-11}$ especially in adenocarcinomas of the breast, ${ }^{6} 11$ colon, or lungs. ${ }^{11}$ Metastases from mammary ${ }^{69}$ and other carcinomas ${ }^{512}$ may also express secretory component. Synthesis of secretory component seems to be independent of tumour differentiation, ${ }^{611}$ except in large bowel carcinomas. ${ }^{7811}$ Secretory component has thus been regarded as a potentially important tumour marker of breast carcinomas. ${ }^{6}$ Furthermore, it has been claimed that secretory component or SIgA in serum reflects the clinical course of breast cancer. ${ }^{13}$ Other disorders, however, may also produce increased serum concentrations of secretory component, particularly liver disease. ${ }^{14-16}$ Secretory component in serum will always be complexed with pIgA and pIgM to form circulating SIgA and SIgM. ${ }^{3}$

Accepted for publication 6 January 1987
As far as we know this is the first study in which serum concentrations of SIgA and SIgM have been determined in relation to other biological variables of breast carcinomas - tumour expression of secretory component, IgA, and carcinoembryonic antigen (CEA); histological grading; tumour cell DNA ploidy; and circulating CEA. Furthermore, our assay reflects the actual molecular state of circulating secretory component and takes into account the different antigenicity of SIgA and SIgM. ${ }^{17}$ To the best of our knowledge previous reports on secretory component in relation to cancer have not considered the clinical relevance of circulating SIgM nor its possible influence on quantitation of SIgA- or secretory : component-in serum.

\section{Material and methods}

The 93 women studied were aged between $20-81$ years and had been admitted for breast tumours. Surgery for breast carcinoma had previously been performed on 15 patients, seven of whom had no recurrence but eight of whom had advanced malignant disease. Of the remaining 78 patients, 48 had carcinomas and 30 benign tumours. Immunohistochemistry, DNA flow cytometry, and serum CEA measurements were performed in 29 of the patients with malignant tumours.

IMMUNOASSAYS FOR CIRCULATING SIgA, SIgM, AND CEA

Serum samples were obtained before surgery and stored at $-70^{\circ} \mathrm{C}$. Differential quantitation of SIgA and SIgM was performed by an enzyme linked 
immunosorbent assay (ELISA), as detailed elsewhere. ${ }^{17}$ Briefly, the assay was based on noncompetitive binding of SIgA and SIgM to microplates coated with an excess of sheep IgG antibodies to human secretory component. Serum concentrations were determined in relation to appropriate SIgA or SIgM standards. Mutual competition between SIgA and SIgM in serum was avoided by testing the samples at sufficiently high dilutions. The concentration of total secretory component in serum was calculated from the SIgA and SIgM values. ${ }^{17}$ Sera from 49 age matched healthy women (aged 20-91 years) were included as controls.

The concentration of CEA in serum was measured by radioimmunoassay. ${ }^{18}$

\section{HISTOLOGICAL GRADING AND}

\section{IMMUNOHISTOCHEMISTRY}

Sections routinely stained with haematoxylin and eosin were used for histological grading according to the World Health Organisation classification. ${ }^{19}$ The immunohistochemical procedure, which has been described previously, ${ }^{20}$ was based on serial sections cut at $6 \mu \mathrm{m}$ from two ethanol fixed paraffin embedded tissue blocks, including representative neoplastic epithelium from each case. Paired immunofluorescence staining performed for secretory component, IgA, and CEA was evaluated semiquantitatively; a score of 3 represented bright overall staining of the tumour epithelium and a score of 0 virtually no staining. The degree of heterogeneous staining pattern was not evaluated in this study.

\section{DNA FLOW CYTOMETRY}

Fresh tumour tissues were studied by quantitation of DNA flow cytometry. The tumours were thus classified as nearly diploid or distinctly aneuploid. The procedure has been described and discussed in detail elsewhere. ${ }^{12}$ Briefly, tumour cell suspensions were stained with ethidium bromide. Flow cytometrical histograms of emission measurements were analysed by planimetry. Lymphocytes from mouse spleen were used as a diploid reference.

\section{STATISTICAL METHODS}

Comparisons between groups were based on the Mann-Whitney U test. Correlations were expressed by Kendall's $\tau$ (taū). All tests were two tailed. Upper reference values for seruin SIgA, SIgM, and total secretory component were taken from those corresponding to the 97.5 percentile (non-parametric) in the control group. ${ }^{21}$

\section{Results}

SERUM CONCENTRATIONS OF

SECRETORY IMMUNOGLOBULINS

Twenty per cent of the patients with either benign or malignant tumours had circulating SIgM concentrations above the upper reference values (fig 1). Raised SIgA concentrations were found in $13 \%$ of the benign and $11 \%$ of the malignant tumours, and nearly all of these serum samples also had raised SIgM concentrations (fig 1). The two patient groups showed significantly (benign tumours, $p=0.02$ ) or probably significantly (malignant tumours, $p=0.07$ ) higher concentrations of total circulating secretory component than the controls. There were no differences in total serum secretory component between those with benign or malignant tumours.

Three of the four patients with carcinoma with the highest total serum concentrations of secretory component were the only ones in this study who had liver metastases indicated in their records: one had liver metastases verified by computed tomography; one developed clinical overt metastases of liver, mediastinum, and spine shortly after surgery (no advanced diagnostic test was performed before surgery to detect

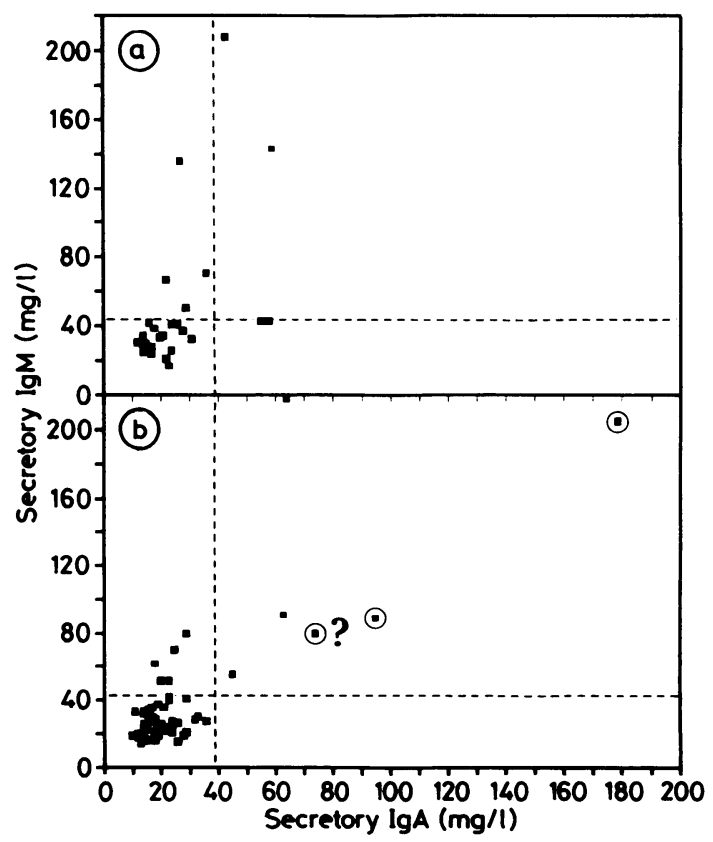

Fig 1 Serum concentration of $\operatorname{SIg} A$ and $\operatorname{SIg} M$ in patients with benign (a) or malignant (b) breast tumours. Dotted lines represent upper normal reference ranges. Circles indicate patients with liver metastases (one questionable). 
liver metastases); and one died three days after admission with disseminated cancer and probable liver disease as judged by increased serum liver enzyme activities (necropsy and other tests were not performed).

The fourth patient with particularly high serum secretory component had localised cancer but was treated for rheumatoid arthritis with gold and naproxene. In contrast, seven patients with carcinoma who had moderately increased serum concentrations of SIgA and SIgM all had localised tumours, and they were otherwise healthy. Among the 45 patients with carcinoma and normal concentrations of circulating secretory component, six had disseminated disease with axillary, pleural, bone, or brain metastases.

The women with benign tumours were generally healthy except for one who was treated for heavy alcohol abuse. She had raised serum concentrations of SIgM (136 mg/l). Of the 17 patients with fibrocystic disease, four had sclerosing adenosis as the dominating histological pattern. These four women had significantly $(p<0.03)$ higher concentrations of circulating secretory component than all the others with benign breast tumours.

\section{IMMUNOHISTOCHEMISTRY FOR SECRETORY}

COMPONENT, IgA, AND CEA

Of the carcinomas that were examined immunohistochemically, $76 \%(\mathrm{n}=22)$ were positive for secretory component (fig 2 ) with predominantly faint staining. Expression of secretory component correlated well with $\operatorname{IgA}$ positivity, but was not related to histopathological grade. Moreover, serum SIgA and SIgM concentrations did not correlate with the expression of secretory component in the tumours; two of five patients with raised serum SIgA or SIgM

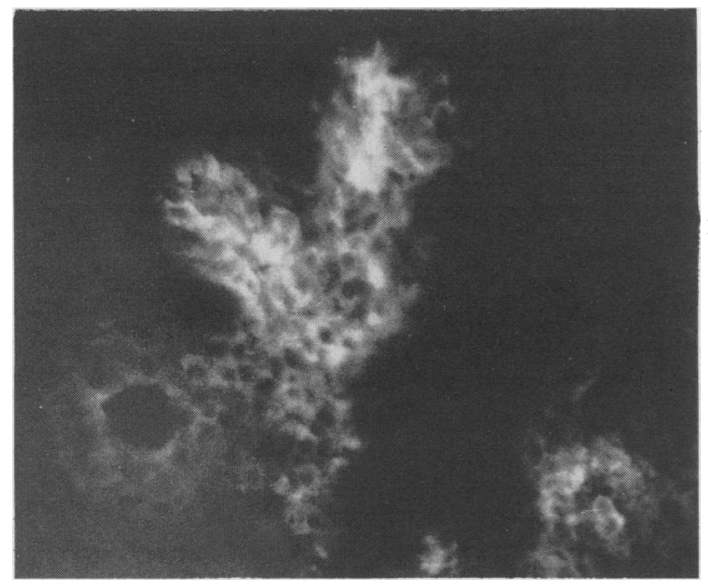

Fig 2 Immunohistochemical staining for secretory component from distinct positive breast carcinoma.

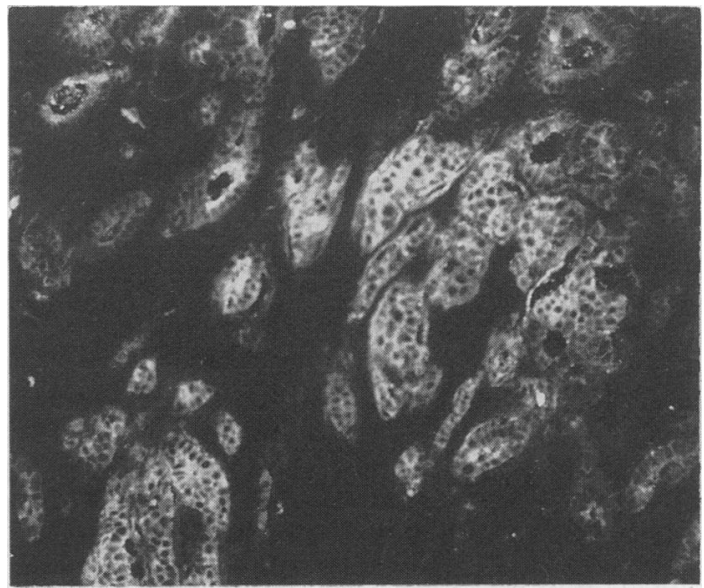

Fig 3 Immunohistochemical staining for $C E A$ from positive breast carcinoma.

concentrations showed no expression of secretory component in the primary tumour.

Most of the carcinomas (55\%) were positive for CEA (fig 3), and $18 \%$ of these patients had raised serum concentrations of CEA. Circulating CEA concentrations showed no significant correlation with tumour CEA expression. Carcinomas with the strongest CEA staining, however, had by far the highest serum CEA concentrations. There was a weak but significant correlation $(\tau=0.34, p<0.02)$ between concentrations of plasma CEA and circulating SIgA and SIgM.

\section{DNA PLOIDY}

When carcinomas were divided according to their DNA ploidy pattern into nearly diploid $(31 \%)$ and aneuploid $(69 \%)$, no significant differences were seen between the two groups in the concentrations of circulating secretory component and CEA, or in tumour expression of secretory component and CEA.

\section{Discussion}

Previous reports have dealt with secretory component as a marker of human breast carcinomas in terms of its expression by primary tumours, ${ }^{61011}$ or metastases, ${ }^{69}$ and its appearance in peripheral blood..$^{131522}$ We found that $76 \%$ of primary breast carcinoma tumours were positive for secretory component, which was well within the range of observations reported by others. ${ }^{610}$

Release of free secretory component (and preformed SIgA) from a tumour could lead to increased circulating SIgA and SIgM, as free secretory component shows high affinity not only for pIgA but par- 
ticularly for pIgM. ${ }^{23}$ Thus free secretory component is always complexed with pIg in serum, either noncovalently (mainly to pIgM) or covalently (mainly to pIgA). ${ }^{3}$ Release of free secretory component alone might be expected to cause preferential increases in SIgM concentration because pIgM is the major circulating pIg. We found that the sensitivity of SIgM as a tumour marker was almost twice that of SIgA.

Twenty per cent of the patients with carcinomas had increased preoperative serum concentrations of SIgM. Other workers have reported $13 \%$ increased SIgA concentrations, ${ }^{15}$ although patients with more advanced disease have shown a higher frequency. ${ }^{1322}$ One of the patients with carcinoma had rheumatoid arthritis, a condition that may cause an increase in serum secretory component. ${ }^{14}$ Interestingly, all three patients with liver disease had particularly high concentrations of circulating secretory component. One had verified liver metastasis and another most likely also had liver disease but died before any specific diagnostic tests could be performed. The third patient developed liver metastases clinically within a short time. Conversely, six additional patients who had disseminated cancer without liver metastases had normal serum values of secretory component.

The possible association between liver metastases and high concentrations of serum secretory component agrees with the findings of previous studies in our laboratory of patients with large bowel carcinomas. ${ }^{24}$ In addition, follow up studies of these patients have shown that our test can detect most patients with liver metastases up to 12 months before the liver metastases become clinically overt (Kvale D, Rognum TO, Brandtzaeg P, unpublished observations). Puleo et al ${ }^{13}$ claimed that total concentrations of secretory component in plasma might reflect the clinical course in metastatic breast cancer, but the greatest increase in secretory component in their study also occurred in patients who developed liver metastases. Other studies ${ }^{1415}$ have reported an association between raised SIgA in serum and metastatic carcinomas with liver disease, although a closer evaluation of tumour properties such as expression of secretory component was not performed. It is impossible, however, to be conclusive on this matter as only three patients with liver metastases were included in our study.

The generally low concentrations of serum SIgA and SIgM in patients with breast carcinoma without liver disease, and the lack of correlation between serum component and tumour expression of secretory component, suggested that the tumours contributed very little of the circulating secretory component. Notably, Stern et al ${ }^{9}$ found lower concentrations of secretory component in cytosol from metastatic mammary carcinomas than from primary tumours.
Metastatic carcinomas may therefore produce less secretory component than that indicated by immunohistochemical observations on tumours positive for secretory component. ${ }^{61011}$ Furthermore, increases in serum secretory component in patients with liver metastases should mainly be ascribed to secondary liver derangements and not to tumour production. ${ }^{24}$

Both from a biological and clinical point of view it seemed justified to include benign breast tumours in a study of breast cancer. We found particularly high concentrations of serum SIgM in patients with sclerosing adenosis in fibrocystic disease. This might reflect the histological and immunohistochemical ${ }^{6}$ features of this condition. The raised SIgM value noted in one alcohol abuser could be due to alcoholic liver disease known to increase the concentrations of circulating $\operatorname{SIg} \mathrm{A}^{716}$ and SIgM. ${ }^{16}$

In conclusion, we measured the concentrations of circulating SIgM and SIgA in patients with benign and malignant breast tumours and found a higher diagnostic sensitivity for SIgM. We did not find any significant correlations between tumour secretory component or CEA expression and the corresponding serum concentrations of secretory component and CEA. Moreover, preoperative concentrations of serum SIgA and SIgM could not indicate the type of lesion. Nevertheless, an association was indicated between high concentrations of circulating secretory component and liver metastases. Liver metastases as an isolated complication in breast cancer, however, is rare. The clinical value of quantitating serum secretory component in relation to this malignancy is therefore questionable.

D Kvale is a research fellow of the Norwegian Cancer Society, and is financially supported by the Norwegian Cancer Society, Nansen's Fund, and Anders Jahre's Fund.

\section{References}

I Mostov KE, Blobel G. A transmembrane precursor of secretory component. J Biol Chem 1982;257:11816-21.

2 Brandtzaeg P, Prydz H. Direct evidence for an integrated function of $\mathbf{J}$ chain and secretory component in epithelial transport of immunoglobulins. Nature (Lond) 1984;311:71-3.

3 Brandtzaeg $\mathbf{P}$. Role of $\mathbf{J}$ chain and secretory component in receptor-mediated glandular and hepatic transport of immunoglobulins in man. Scand J Immunol 1985;22:111-46.

4 Brandtzaeg $P$. The secretory immune system of lactating human mammary glands compared with other exocrine organs. Ann N Y Acad Sci 1983;409:353.

5 Gotoh T, Takishita Y, Doi H, Tsubura E. Secretory-componentproducing lung cancer with hypergammaglobulinemia of secretory IgA. Cancer 1981;48:1776-82.

6 Harris JP, South MA. Secretory component; A glandular epithelial cell marker. Am J Pathol 1981;105:47-53.

7 Poger ME, Hirsch BR, Lamm ME. Synthesis of secretory component by colonic neoplasms. Am J Pathol 1976;82:327-38.

8 Rognum TO, Elgjo K, Brandtzaeg P, Ørjasaether H, Bergan A. 
Plasma carcinoembryonic antigen concentrations and immunohistochemical patterns of epithelial marker antigens in patients with large bowel carcinoma. J Clin Pathol 1982;35:922-33.

9 Stern JE, Underdown BJ, Crichlow RW, Wira CR. Secretory component in breast cancer. Analysis of the levels in primary and metastatic disease. Cancer Immunol Immunother 1985;19:226-30.

10 Walker RA. Differentation of human breast carcinomas: An immunohistological study of appropriate and inappropriate protein production. $J$ Pathol 1981;135:87-95.

11 Brooks JJ, Ernst CS. Immunoreactive secretory component of IgA in human tissues and tumors. Am J Clin Pathol 1984;82:660-5.

12 Rognum TO, Thorud E, Brandtzaeg P. Preservation of cytometric DNA distribution and epithelial marker expression after tumor progression of human large bowel carcinomas. Cancer 1985;56:1658-66.

13 Puleo EAT, Haagensen DE, Dawson JR, Gall SA. Study of secretory component in patients with metastatic breast cancer. Am J Obstet Gynecol 1979;134:899-902.

14 Delacroix D, Vaerman JP. Reassessment of levels of secretory IgA in pathological sera using a quantitative radioimmunoassay. Clin Exp Immunol 1981;43:633-40.

15 Homburger HA, Casey M, Jacob GL, Klee GG. Measurements of secretory IgA by radioimmunoassay in patients with chronic non-alcoholic liver disease or carcinoma. Am J Clin Pathol 1984;81:569-74.

16 Kvale D, Schrumpf E, Brandtzaeg P, Solberg HE, Fausa O, Elgjo $\mathrm{K}$. Circulating immunoglobulins of the $A$ and $M$ isotypes in chronic liver disease. $J$ Hepatol (in press).

17 Kvale D, Brandtzaeg P. An enzyme-linked immunosorbent assay for differential quantitation of secretory immunoglobulins of the $\mathbf{A}$ and $\mathbf{M}$ isotypes in human serum. $J$ Immunol Methods 1986;86:107-14.

18 Børmer O. A direct assay for carcinoembryonic antigen in serum and its diagnostic value in metastatic breast cancer. Clin Biochem 1982:15:128-32.

19 Scarff RW, Torloni H. Histological typing of breast tumors. In: International classification of tumors No 2. Geneva: World Health Organisation, 1968:19-20.

20 Rognum TO, Brandtzaeg P, Ørjasaether H, Elgjo K, Hognestad J. Immunohistochemical study of secretory component, secretory $\operatorname{IgA}$ and carcinoembryonic antigen in large bowel carcinomas. Pathol Res Pract 1980;170:126-45.

21 Solberg HE. The theory of reference values, part 5. J Clin Chem Clin Biochem 1983;21:749-60.

22 LoGerfo P, McLanahan S. Serum secretory IgA levels in patients with neoplastic disease. J Surg Res 1976;20:481-4.

23 Brandtzaeg P. Human secretory component-VI. Immunoglobulin-binding properties. Immunochemistry 1977;14: 179-88.

24 Kvale D, Rognum TO, Brandtzaeg P. Elevated levels of secretory immunoglobulins $\mathbf{A}$ and $\mathbf{M}$ in serum of patients with large bowel carcinoma indicate liver metastases. Cancer 1987;59: 203-7.

Requests for reprints to: Dr D Kvale, LIIPAT, Institute of Pathology, Rikshospitalet, N-0027 Oslo 1, Norway. 
The Design and Analysis of Long-Term Animal Experiments. Statistical Methods in Cancer Research. Vol. III. IARC Scientific Publications No. 79. JJ Gart, D Krewski, PN Lee, RE Tarone, J Wahrendorf. (Pp. 219; £28.) Oxford University Press. 1987. ISBN 9283211798

Most attempts to understand and control industrial or domestic exposure to chemicals and radiation as the causes of cancer, and equally, much research into the stages of carcinogenesis are based on long term experiments in animals. The design and analysis of such studies is far more complex than is often realised, but if the work is to be worthwhile, extraneous factors must be rigorously controlled and the results must be evaluated by appropriate statistical procedures.

The introduction in 1972 of a practical method of actuarial analysis of carcinogenicity tests was a great advance in this difficult field. The originators of that statistical technique have now combined with others to write a first class account of how to do and analyse such experiments. They and their sponsor, the IARC, are to be congratulated on a lucid account which shows experimentalists what to do in simple terms, and which separately presents the statistical theory on which the practical procedures are based.

The results of long term experiments in animal and clinical research and for regulation govern most of our usage of chemicals. This book needs to be read and understood by every scientist and administrator concerned with cancer and its possible causes.

AD DAYAN

\section{Notices}

\section{The International Symposium BIOTECH Ria 88}

\section{Molecular probes: technology and medical applications}

Florence (Italy), at the Congress Palace, April 11-13, 1988

List of the sessions: molecular probes in genetic diseases; molecular probes in oncology; molecular probes in infectious diseases.

For further information please contact the organising secretariat: Fondazione Giovanni Lorenzini, Via Monte Napoleone, 23-20121 Milan (Italy).

\section{Joint Royal College of Surgeons/Imperial Cancer Research Fund Histopathology Unit}

As part of this unit, which has been established for the purpose of advancing the science of diagnostic histopathology, a panel of experienced histopathologists provides advice on diagnostic problems to any pathologist seeking a second opinion. Material can be sent as wet tissue, fixed tissue, paraffin blocks, or sections. If stained sections are submitted additional unstained sections should be sent whenever possible. Where blocks are sent, they will be returned in due course when the sender indicates that this is necessary. Special arrangements can be made, where possible, for the examination of frozen sections or material requiring electron microscopy. There is no charge for these services.

The Panel is now in its third year and has dealt with an average of between four and five cases per week. These have come from more than $\mathbf{5 0}$ different hospitals in the United Kingdom and from 10 different countries. The membership of the panel at present includes: Professor NFC Gowing, Professor DH Mackenzie, Dr BC Morson, Dr RCB Pugh, Professor H Spencer, Dr AG Stansfeld, Professor AC Thackray and Dr KAD Turk. The work of the panel is being coordinated by Professor B Cohen from whom request forms can be obtained and to whom enquiries can be addressed at: the Histopathology Unit, 35-43 Lincoln's Inn Fields, London WC2A 3PN (01-242 0200).

\section{ASSOCIATION OF CLINICAL PATHOLOGISTS}

JUNIOR MEMBERSHIP

Junior membership of the Association is available to all trainees in pathology for up to six years after the start of training. The annual subscription is $£ 15$ and may be claimed against tax. All junior members receive copies of the Journal of Clinical Pathology. Other benefits include membership of the Junior Members' Group and a regular junior members' newsletter; the ACP Newsletter and all other documents regularly sent to full members including the postgraduate education programme.

Apply to: Dr PP Anthony, Education Secretary, Postgraduate Medical School, Barrack Road, Exeter EX2 SDW, Devon.
West of Scotland Committee for Postgraduate Medical Education

Five day course on histopathology of the skin

21-25 March 1988

This course is designed to meet the needs of both pathologists and dermatologists with an interest in dermatopathology. Both newcomers to the field and those with considerable experience of skin pathology will find the course of value.

The course, which is organised by Professor R M MacKie, is divided into three sections: lecture discussions, individual microscopy sessions, and projection sessions, at which material submitted by those attending the course will be demonstrated and discussed.

The syllabus covered includes the structure of normal skin, congenital skin disease, inflammatory dermatoses, granulomata and viral disease, cutaneous reticuloses, skin tumours, bullous diseases and connective tissue problems including vasculitis.

The course will be held in the University Department of Dermatology, Anderson College Building, Western Infirmary, Glasgow G11 6NT, and accommodation will be available at a nearby Hall of Residence.

Course Fee $£ 110$

Further information and application forms from: Mrs W E Scott, administrative assistant, West of Scotland Committee for Postgraduate Medical Education, University of Glasgow, GLASGOW G12 8QQ.

\section{Corrections}

An error occurred in the summary of $J$ Clin Pathol 1987;40:879-84. (Barbatis et al.) The last paragraph should read: Bile duct epithelium expresses HLA-DR in primary biliary cirrhosis, large duct obstruction and drug induced cholestasis indicating that HLA-DR expression in bile duct epithelium is not exclusive to primary biliary cirrhosis.

(See also correspondence from Professor Wright)

In the paper by Kvale D et al. ( $J$ Clin Pathol 1987;40:621-5), the first line of the summary should read: The serum concentrations of IgA and IgM associated secretory component ... and not serum concentrations of IgAp and IgMr.... 